\title{
AUTOCUIDADO DEL PACIENTE CON HIPERTENSIÓN ARTERIAL Y SU RELACIÓN CON LOS FACTORES INDIVIDUALES Y SOCIOCULTURALES EN PACIENTES ATENDIDOS EN UN HOSPITAL DE HUANCAYO.
}

\section{" \\ PATIENT SELFCARE WITH ARTERIAL HYPERTENSION AND ITS RELATION TO SOCIOCULTURAL AND INDIVIDUAL FACTORS IN PATIENTS WHO WERE TAKEN CARE OF IN A HUANCAYO HOSPITAL}

\section{AUTORES: Suasnabar Cueva Edith ${ }^{1}$, Tello Carhuanca Rebeca², Villegas Félix Teresa Leonor ${ }^{3}$, Huamancaja} Padilla Elizabeth ${ }^{4}$.

\section{RESUMEN}

OBJETIVO: Determinar la práctica de autocuidado y su relación con los factores individuales y socioculturales en pacientes hipertensos atendidos en un hospital de Huancayo.

MÉTODO: Estuvo conformada por 50 pacientes atendidos en el programa de hipertensión de un hospital de la ciudad de Huancayo. Esta investigación fue descriptivo-correlacional, no experimental.

RESULTADOS: Se encontró que los niveles de autocuidado de los pacientes que participaron en el estudio se caracterizan por tener un nivel Medio $(68,00 \%)$, sólo el $22,00 \%$ presentó un nivel Alto y el $10,00 \%$ tuvo un nivel Bajo. En relación a los factores individuales se encontró un nivel Alto $(48,00 \%)$ y un nivel Medio (42,00\%). Los niveles de factores socioculturales de los pacientes hipertensos fueron Alto $(50,00 \%)$, Medio $(44,00 \%)$ y sólo una minoría $(6,00 \%)$ tuvo un nivel Bajo. Estos resultados nos permitieron concluir que: Existe una relación directa y significativa entre el autocuidado y los factores individuales y socioculturales de los pacientes hipertensos del programa de hipertensión de un hospital de Huancayo, para un nivel de significación de $a=0,05$.

PALABRAS CLAVES: Hipertensión arterial, factores individuales, factores socioculturales.

\section{ABSTRACT \\ OBJECTIVE: To determine the practice of self and its relation to individual and sociocultural factors in hypertensive patients treated at a hospital in Huancayo.}

METHOD: Consisted of 50 patients treated at the hypertension program at a hospital in the city of Huancayo. This study was descriptive-correlational, not experimental.
RESULTS: We found that the levels of self-care of patients who participated in the study are characterized as having an average level (68.00\%), only $22.00 \%$ had a high level and $10.00 \%$ had a level Low. Regarding individual factors High level (48.00\%) and means $(42,00 \%)$ level was found. Sociocultural levels of hypertensive patients were Alto (50.00\%), Medium (44.00\%) and only a minority $(6.00 \%)$ had a low level. These results allowed us to conclude that: There is a direct and significant relationship between the self and the individual and sociocultural factors of hypertensive patients in the hypertension program at a hospital in Huancayo, for a significance level of $a=0.05$.

KEYWORDS: Hypertension, individual factors, sociocultural factors.

\section{INTRODUCCIÓN}

Autocuidado es la capacidad que posee cada individuo de desarrollar conductas destinadas a mantener su salud, estas conductas son llevadas a cabo por sí mismo en el marco del conocimiento. El problema de las enfermedades crónicas afecta en términos generales a la población en el mundo entero y entre éstas la hipertensión arterial, problema de salud pública tanto en nuestro país como en otros lugares. Por otro lado el reconocimiento de la existencia de factores individuales y socioculturales en los pacientes con hipertensión arterial, tendrían que ver con las prácticas de autocuidado.

A nivel local y regional un hospital en Huancayo brinda servicios de salud que incluyen el diagnóstico, el tratamiento, control periódico y la educación sobre el autocuidado en esta enfermedad crónica, atención que sólo cubre a un determinado grupo poblacional, por lo tanto, como se menciona líneas 
arriba, entendiendo el autocuidado por prácticas que lleva a cabo el individuo para cuidar su salud y evitar complicaciones, habiendo sido informado, se esperaría que el paciente aplique estos conocimientos y por lo tanto se reduzca la tasa de complicaciones y muertes como consecuencia de esta enfermedad. Sin embargo esto no sucede, por muchos factores, entre ellos los factores individuales y socioculturales, de allí el interés de este estudio de conocer la relación existente entre estas variables.

El presente trabajo de investigación surgió a través de la observación que se realizó a los pacientes hipertensos atendidos en el programa de hipertensión de un hospital de Huancayo ya que muchas veces llegaban con ciertas complicaciones por no aplicar los cuidados que debían practicar para evitar estas complicaciones, pese a haber participado de programas de educación, lo que nos llevó a pensar que existen además otros factores tanto individuales como socioculturales que podrían estar relacionados con el autocuidado; en este sentido el estudio realizado tuvo el objetivo de determinar la práctica de autocuidado y su relación con los factores individuales y socioculturales en pacientes hipertensos atendidos en un hospital de Huancayo.

\section{MATERIAL Y MÉTODOS}

En cuanto al diseño, esta investigación es descriptivocorrelacional, no experimental, la población estuvo conformada por todos los pacientes hipertensos que son atendidos en el programa de control de hipertensión arterial de un hospital de Huancayo. El muestreo fué no probabilístico y casual fueron captados después de ser atendidos en el programa de hipertensión arterial, la muestra estuvo conformada por 50 pacientes. El instrumento utilizado fue Cuestionario. Se aplicó a los 50 pacientes hipertensos que fueron atendidos en el programa de control de hipertensión arterial de un hospital de Huancayo.

El tratamiento estadístico se realizó mediante la aplicación del SOFTWARE PASW Versión 21, donde se utilizaron los estadígrafos descriptivos y de la estadística inferencial para la correspondiente prueba de hipótesis.

\section{RESULTADOS}

En el presente capítulo se muestran los resultados de la aplicación de la encuesta a los 50 pacientes que se atendieron en el Programa de hipertensión de un hospital de Huancayo, sobre los datos generales, el autocuidado de los pacientes, los factores individuales y socioculturales.

TABLA 1: DESCRIPCIÓN DE LOS

DATOS GENERALES DE LA MUESTRA DE INVESTIGACIÓN

\begin{tabular}{lcc}
\hline & $\mathbf{f i}$ & $\%$ \\
\hline Muestra & 50 & 100,00 \\
Edad del paciente & & \\
Promedio & 43,92 & \\
DS & 15,73 & \\
C.V.(\%) & & $35,82 \%$ \\
Género del paciente & & \\
Femenino & 31 & 62,00 \\
Masculino & 19 & 38,00 \\
Estado de desarrollo & & \\
Adulto joven & 34 & 68,00 \\
Adulto mayor & 16 & 32,00 \\
Senescencia & 0 & 0,00 \\
\hline Leyenda: & & \\
fil: :recuencia de casos & & \\
DS: Desviación estándar \\
C.V.(\%): Coeficiente de variabilidad
\end{tabular}

En la tabla No 1 se observa que la edad promedio de la muestra de pacientes que participan en la investigación es de 43,92 años, con una desviación de 15,73 años, lo que permite afirmar que la edad de los pacientes son heterogéneas (35,82\%). El $62,00 \%$ de los pacientes de la muestra de estudio son del género femenino y representan la mayoría, mientras que el $38,00 \%$ son del género masculino. El estado de desarrollo de los pacientes, en su mayoría $(68,00 \%)$ son adultos jóvenes, el $32,00 \%$ son adultos mayores y ningún paciente es de la etapa de senescencia. 
TABLA 2: FACTORES DE LOS PATRONES DE VIDA

\begin{tabular}{lcc}
\hline & $\mathbf{f i}$ & $\mathbf{\%}$ \\
\hline Consumo de cigarrillos & $\mathbf{5 0}$ & $\mathbf{1 0 0 , 0 0}$ \\
$\mathrm{Si}$ & 9 & 18,00 \\
No & 41 & 82,00 \\
Realiza ejercicios $\mathbf{3 0} \mathbf{m i n}$. & & \\
$\mathrm{Si}$ & 24 & 48,00 \\
No & 26 & 52,00 \\
Consumo de bebidas alcohólicas & & \\
$\mathrm{Si}$ & 17 & 34,00 \\
No & 33 & 66,00 \\
Consumo de mucha sal & & \\
Si & 11 & 22,00 \\
No & 39 & 78,00 \\
Consumo de frutas & & \\
Si & 32 & 64,00 \\
No & 18 & 36,00 \\
Consumo de verduras & & \\
Si & 37 & 74,00 \\
No & 13 & 26,00 \\
Si & & \\
No & 29 & 58,00 \\
\hline Leyenda: fi: Frecuencia & 21 & 42,00 \\
\hline
\end{tabular}

Se observa, en la tabla No 2, las frecuencias de los patrones de vida de los 50 pacientes que participan en la investigación, donde que la mayoría $82,00 \%$ (41) de los pacientes que participan en la investigación no consumen cigarrillos y sólo el $18,00 \%$ (9) de los pacientes si consume cigarrillos; el $48,00 \%$ de los pacientes realizan ejercicios de 30 minutos tres veces por semana, la mayoría $(52,00 \%)$ no hace ejercicios; el $34,00 \%$ (17), de los pacientes consume bebidas alcohólicas, mientras que la mayoría $(66,00 \%)$ no consume; el $22,00 \%$ de los pacientes agrega más sal a sus alimentos y mayoría $(78,00 \%)$ no consume sal en exceso; la mayoría $(64,00 \%)$ de los pacientes consume frutas diariamente pero el $36,00 \%$ no consume; el $74,00 \%$ de los pacientes consumen verduras diariamente, pero una minoría $(26,00 \%)$ no lo hace; el $58,00 \%(29)$ de los pacientes consumen alimentos ricos en fritos o grasas y constituyen la mayoría, mientras que una minoría $(42,00 \%)$ no consume.

\section{TABLA 3: ESTADÍGRAFOS Y NIVELES DE LOS FACTORES DE LOS PATRONES DE VIDA DE LOS PACIENTES}

\begin{tabular}{lcr}
\hline Patrones de vida & $\mathbf{f i}$ & \% \\
\hline $\begin{array}{l}\text { Muestra: } \\
\text { Puntajes }\end{array}$ & 50 & $100,00 \%$ \\
Promedio & & \\
DS & 4,54 & \\
C.V.(\%) & 1,64 & \\
Niveles & & $36,12 \%$ \\
Bajo & 3 & \\
Medio & 23 & $6,00 \%$ \\
Alto & 24 & $48,00 \%$ \\
\hline
\end{tabular}

\section{Leyenda:}

fi: Frecuencia de casos

DS: Desviación estándar

C.V.(\%): Coeficiente de variabilidad

En la tabla No 3 se observa que el puntaje promedio obtenido en los patrones de vida es 4,54 puntos en la escala de 0 a 7 puntos, con una desviación de 1,64 y un coeficiente de variabilidad (36,12\%) que caracteriza a los puntajes como heterogéneas. Los niveles de los patrones de vida de los 50 pacientes que participan en el estudio se caracterizan por tener un nivel Alto $(48,00 \%)$ y un nivel Medio $(46,00 \%)$.

En la tabla No 4, se observa que la mayoría $76,00 \%$ (38) de los pacientes viven en la zona urbana, mientras que sólo el $24,00 \%$ vive en zona rural; el $64,00 \%$ (32) de los pacientes manejan altos niveles de estrés y el $36 \%$ no lo hace; la mayoría $(70,00 \%)$ de los pacientes no busca relajarse cuando tiene estrés y la minoría $(30,00 \%)$ si lo hace; la mayoría $(54,00 \%)$ de los pacientes no duermen las 8 horas diarias, mientras que el $46,00 \%$ de los pacientes si lo hace. 
TABLA 4: FACTORES DEL AMBIENTE

\begin{tabular}{lcc}
\hline & $\mathbf{f i}$ & $\mathbf{\%}$ \\
\hline Zona donde vive & $\mathbf{5 0}$ & $\mathbf{1 0 0 , 0 0}$ \\
Rural & 12 & 24,00 \\
Urbano & 38 & 76,00 \\
Maneja de altos niveles de estrés & 32 & 64,00 \\
Si & 18 & 36,00 \\
No & & \\
¿Busca relajarse? & 15 & 30,00 \\
Si & 35 & 70,00 \\
No & & \\
Duerme 8 horas diarias & 23 & 46,00 \\
Si & 27 & 54,00
\end{tabular}

Leyenda: fi: Frecuencia

TABLA 5: ESTADÍGRAFOS Y NIVELES DEL AMBIENTE

\begin{tabular}{lcc}
\hline Factores del ambiente & $\mathbf{f i}$ & $\mathbf{\%}$ \\
\hline Muestra: & 50 & $100,00 \%$ \\
Puntajes & & \\
Promedio & 1,36 & \\
DS & 1,14 & \\
C.V.(\%) & & $83,82 \%$ \\
Niveles & & \\
Bajo & 30 & $60,00 \%$ \\
Medio & 18 & $36,00 \%$ \\
Alto & 2 & $4,00 \%$ \\
\hline
\end{tabular}

Leyenda: fi: Frecuencia de casos

Se observa, en la tabla No 5 que el puntaje promedio obtenido en los factores del ambiente es de 1,36 puntos en la escala de 0 a 4 puntos, con una desviación de 1,14 y un coeficiente de variabilidad $(83,82 \%)$ que indica que los puntajes son heterogéneos. Los niveles de los factores del ambiente de los pacientes en su mayoría $(60,00 \%)$ es Bajo y sólo el $36,00 \%$ de los pacientes presenta un nivel Medio.

TABLA 6: ESTADÍGRAFOS Y NIVELES DE LA VARIABLE AUTOCUIDADO DE LOS PACIENTES

\begin{tabular}{|c|c|c|}
\hline Patrones de vida & fi & $\%$ \\
\hline Muestra: & 50 & $100,00 \%$ \\
\hline \multicolumn{3}{|l|}{ Puntajes } \\
\hline Promedio & 5,90 & \\
\hline DS & 2,21 & \\
\hline C.V.(\%) & & $37,46 \%$ \\
\hline \multicolumn{3}{|l|}{ Niveles } \\
\hline Bajo & 5 & $10,00 \%$ \\
\hline Medio & 34 & $68,00 \%$ \\
\hline Alto & 11 & $22,00 \%$ \\
\hline
\end{tabular}

En la tabla No 6 se observa que el puntaje promedio obtenido en la variable autocuidado es de 5,90 puntos en la escala de 0 a 11 puntos, con una desviación de 2,21 y un coeficiente de variabilidad (37,46\%) que caracteriza a los puntajes como heterogéneos. Los niveles del autocuidado de los pacientes que participan en el estudio se caracterizan por tener un nivel Medio $(68,00 \%$ ), ya que sólo el $22,00 \%$ presenta un nivel Alto y el $10,00 \%$ tiene un nivel Bajo. 
TABLA 7: FACTORES INDIVIDUALES

\begin{tabular}{lcc}
\hline & $\mathbf{f i}$ & $\mathbf{\%}$ \\
\hline Estado de desarrollo & $\mathbf{5 0}$ & $\mathbf{1 0 0 , 0 0}$ \\
Etapa adulto joven & 34 & 68,00 \\
$\begin{array}{l}\text { Etapa adulto mayor } \\
\text { Etapa senescencia }\end{array}$ & 16 & 32,00 \\
Estado de salud & 0 & \\
Malo & & \\
Regular & 7 & 14,00 \\
Bueno & 8 & 16,00 \\
$\begin{array}{l}\text { Excelente } \\
\text { Indice de Masa Corporal }\end{array}$ & 19 & 38,00 \\
Normal & 16 & 32,00 \\
Sobrepeso & & \\
Antecedentes de enfermedad de los & 30 & 60,00 \\
parientes & 20 & 40,00 \\
$\begin{array}{l}\text { Si } \\
\text { No }\end{array}$ & & \\
Recursos económicos & 25 & 50,00 \\
Bajo (0 a S/.799) & 25 & 50,00 \\
Medio (S/.800 a S/.1199) & & \\
Alto (S/.1200 a más) & 5 & 10,00 \\
\hline
\end{tabular}

Leyenda: fi: Frecuencia

Se observa, en la tabla No 7, las frecuencias de los indicadores de la variable Factores individuales, donde que la mayoría $68,00 \%$ (34) de los pacientes que participan en la investigación están en la etapa de adulto joven, el $16,00 \%$ se encuentran en la etapa de adulto mayor; el $38,00 \%$ de los pacientes tienen un estado de salud Bueno, el $32,00 \%$ presenta un estado de salud Excelente, el $16,00 \%$ se encuentra Regular y sólo el $14,00 \%$ tiene un estado de salud Malo; la mayoría (60,00\%) de los pacientes tiene un Índice de Masa Corporal (IMC) Normal y el $40,00 \%$ presenta un IMC con sobrepeso; la mitad $(50,00 \%)$ de pacientes tienen antecedentes de enfermedades en sus parientes cercanos, mientras que la otra mitad (50,00\%) no tiene; el $48,00 \%$ de los pacientes tienen un nivel de recursos económicos familiares mensuales Alto (deS/. 1200 a más), el 42,00\% de los pacientes presenta un nivel Medio (de S/. 800 a S/. 1199) y sólo el 10,00\% tienen un nivel Bajo (de S/. 0 a S/. 799).

TABLA 8: ESTADÍGRAFOS Y NIVELES DE LOS FACTORES INDIVIDUALES

\begin{tabular}{lcc}
\hline Patrones de vida & $\mathbf{f i}$ & $\mathbf{\%}$ \\
\hline Muestra: & 50 & $100,00 \%$ \\
Puntajes & & \\
Promedio & 9,04 & \\
DS & 1,91 & \\
C.V.(\%) & & $21,13 \%$ \\
Niveles & 5 & \\
Bajo & 21 & $40,00 \%$ \\
Medio & 24 & $48,00 \%$ \\
Alto & & \\
\hline Leyenda: & & \\
fi: Frecuencia de casos \\
DS: Desviación estándar \\
C.V.(\%): Coeficiente de variabilidad
\end{tabular}

En la tabla No 8 se observa que el puntaje promedio obtenido en los factores individuales es de 9,04 puntos en la escala de 0 a 9 puntos, con una desviación de 1,91 y un coeficiente de variabilidad $(21,13 \%)$ que caracteriza a los puntajes como homogéneos. Los niveles de los factores individuales de los pacientes se caracterizan por tener un nivel Alto $(48,00 \%)$ y un nivel Medio $(42,00 \%)$. 
TABLA 9: FACTORES SOCIOCULTURALES

\begin{tabular}{lcr}
\hline & $\mathbf{f i}$ & $\mathbf{\%}$ \\
\hline Grado de instrucción & $\mathbf{5 0}$ & $\mathbf{1 0 0 , 0 0}$ \\
$\begin{array}{l}\text { Analfabeto } \\
\text { Primaria incompleta }\end{array}$ & 3 & 6,00 \\
Primaria completa & 6 & 12,00 \\
Secundaria incompleta & 6 & 12,00 \\
Secundaria completa & 3 & 6,00 \\
Técnico & 4 & 8,00 \\
Universitario & 12 & 24,00 \\
Pertenencia a grupo de interacción & 16 & 32,00 \\
Social & & \\
Si & 17 & 34,00 \\
No & 33 & 66,00 \\
Estado civil & & \\
Soltero/separado & 18 & 36,00 \\
Casado/conviviente & 32 & 64,00 \\
Tiene apoyo familiar para cuidar salud & & \\
Si & 31 & 62,00 \\
No & 19 & 38,00 \\
Buenas relaciones familiares & & \\
Si & 43 & 86,00 \\
No & 7 & 14,00 \\
Preocupación familiar & & \\
Si & 39 & 78,00 \\
No & 11 & 22,00 \\
\hline Leyenda: fi: Frecuencia & & \\
\hline
\end{tabular}

En la tabla No 9, se observa las frecuencias de los indicadores de la variable Factores socioculturales, donde que la mayoría $32,00 \%$ (16) de los pacientes que participan en la investigación tienen estudios universitarios, el $24,00 \%$ tienen estudios técnicos, el $24,00 \%$ de los pacientes tienen estudios de educación primaria completa o incompleta, el $14,00 \%$ tienen estudios de educación secundaria completa o incompleta y sólo el $6 \%$ de los pacientes son analfabetos; la mayoría $(66,00 \%)$ de los pacientes pertenecen a grupos de interacción social y el $34,00 \%$ no; el $64,00 \%$ de los pacientes están casados o son convivientes, mientras que $36,00 \%$ son solteros o se han separado de sus respectivas parejas; el $62,00 \%$ de los pacientes tienen apoyo para cuidar su salud, el $38,00 \%$ no lo tiene; el $86,00 \%$ de los pacientes mantienen buenas relaciones con sus familiares y una minoría $(14,00 \%)$ no; el $78,00 \%$ de los pacientes entrevistados tienen familiares que se preocupan por su salud mientras que el $22,00 \%$ de los pacientes no cuentan con la preocupación de sus familiares.

En la tabla No 10 se observa que el puntaje promedio obtenido en los factores socioculturales es de 8,22 puntos en la escala de 0 a 12 puntos, con una desviación de 2,82 puntos y un coeficiente de variabilidad (34,31\%) que caracteriza a los puntajes como heterogéneas. Los niveles de los factores socioculturales de los pacientes se caracterizan por tener un nivel Alto $(50,00 \%)$ y un nivel Medio $(44,00 \%)$, ya que solo una minoría $(6,00 \%)$ tienen un nivel Bajo en los factores socioculturales.

TABLA 10: ESTADÍGRAFOS Y NIVELES DE LOS FACTORES SOCIOCULTURALES

\begin{tabular}{lcr}
\hline Patrones de vida & $\mathbf{f i}$ & \multicolumn{1}{c}{$\%$} \\
\hline $\begin{array}{l}\text { Muestra: } \\
\text { Puntajes }\end{array}$ & 50 & $100,00 \%$ \\
Promedio & & \\
DS & 8,22 & \\
C.V.(\%) & 2,82 & \\
Niveles & & $34,31 \%$ \\
Bajo & 3 & \\
Medio & 22 & $44,00 \%$ \\
Alto & 25 & $50,00 \%$ \\
\hline $\begin{array}{l}\text { Leyenda: } \\
\text { fi: Frecuencia de casos }\end{array}$ & & \\
DS: Desviación estándar \\
C.V.(\%): Coeficiente de variabilidad
\end{tabular}




\section{RELACIÓN ENTRE LAS VARIABLES DE INVESTIGACIÓN} TABLA 11: RELACIÓN ENTRE LAS VARIABLES DE INVESTIGACIÓN

\begin{tabular}{|c|c|c|}
\hline & Autocuidado & \\
\hline Factores & $\begin{array}{c}\text { Correlación } \\
\text { Pearson }\end{array}$ & p-valor \\
\hline Individuales & $0,457^{\star *}$ & 0,000 \\
\hline Socioculturales & $0,664^{* *}$ & 0,000 \\
\hline Individuales y socioculturales & $0,647^{* *}$ & 0,000 \\
\hline
\end{tabular}

Se presenta, en la tabla No 11, los coeficientes de correlación de Pearson (r) entre las variables de investigación: Se observa que la variable Autocuidado de los pacientes y la variable Factores individuales se correlacionan de manera directa y significativa, porque el coeficiente de Pearson $(0,457)$ es positivo, y como el $p$-valor $=0,000$ es menor al $1 \%(0,010)$ entonces se afirma que la correlación es significativa. También se aprecia que el coeficiente de correlación de Pearson $(0,664)$ entre las variables Autocuidado y Factores socioculturales es positivo para un $p$-valor $=0,000$ por lo que se afirma que dichas variables se relacionan de manera directa y significativa, para un nivel de confianza del $99 \%$. Demás se observa que la variable Autocuidado y las variables Factores individuales y factores Socioculturales se correlacionan de manera directa y significativa, ya que el coeficiente de Pearson $(0,647)$ es positivo, y como el $p$-valor $=0,000$ es menor al $1 \%(0,010)$.

Conclusión estadística: Se demuestra que: Existe una relación directa y significativa entre el autocuidado y los factores individuales y socioculturales de los pacientes hipertensos del programa de hipertensión arterial de un hospital de Huancayo, para un nivel de significación de a=0,05

\section{DISCUSIÓN}

En el presente trabajo de investigación se observa que la edad promedio de la población en estudio es de 43.92 años, el $62 \%$ son del género femenino y en cuanto al estado de desarrollo de los pacientes el $68 \%$ son adultos jóvenes que en su mayoría aplican medidas de autocuidado, datos que coinciden con lo señalado por Vásquez en su estudio titulado "Autocuidado para el manejo de Hipertensión Arterial", en el que el $45 \%$ de la población es adulta joven y el $66 \%$ de sexo femenino.

Por otro lado en relación a la variable autocuidado, Intriago V., y Mevéndez J., realizaron el trabajo de investigación: "Autocuidado de pacientes hipertensos del Club de Corazones Valientes del Hospital Naval de Guayaquil. El estudio permitió reconocer las falencias y problemas que existe en el club al no evidenciarse una aplicación correcta del autocuidado en los pacientes hipertensos, llegando a la conclusión de que la hipertensión arterial, al no ser controlada ni tratada puede provocar complicaciones graves e inclusive la muerte, por lo que al encontrar dificultades en relación a la aplicación del autocuidado en este grupo de pacientes. Por el contrario, en nuestro estudio encontramos un mayor porcentaje de pacientes que aplican medidas de autocuidado, el $48 \%$ tiene un nivel alto y $46 \%$ un nivel medio, por ejemplo, en cuánto a patrones de vida de los 50 pacientes que participan en el estudio, el $82 \%$ de la población no fuma, el $48 \%$ de los pacientes realizan ejercicios de 30 minutos tres veces por semana, el $66 \%$ no consume bebidas alcohólicas, el $78 \%$ no consume sal en exceso y el $64 \%$ consume verduras diariamente, por lo que en relación al estudio mencionado, donde no se evidencia la aplicación del autocuidado, en el presente estudio queda establecido que los niveles de autocuidado de los pacientes que participaron en el estudio se caracterizan por tener un nivel Medio(68\%), sólo el $22 \%$ presenta un nivel Alto y el $10 \%$ tiene un nivel Bajo, confirmando lo encontrado por Fanarraga J. en su tesis titulada " Conocimiento y Práctica de Autocuidado Domiciliario en Pacientes Adultos con Hipertensión Arterial en Consultorios Externos de Cardiología del Hospital GullermoAlmenara Irigoyen Essalud", que señala en cuánto a la práctica de autocuidado el $69 \%$ se controlan la presión arterial, el $77 \%$ realizan caminatas durante el día y el $87 \%$ tienen una alimentación adecuada conteniendo frutas y verduras.

Colman J., en su trabajo de investigación titulado "Participación del profesional de enfermería en la promoción de hábitos de salud y el autocuidado destinadosa prevenircomplicaciones porhipertensión arterial en Paraguay, señala que los resultados de su investigación indican que los profesionales de enfermería que prestan asistencia sanitaria en el Hospital Regional de Caazapá participan activamente en la promoción de hábitos de salud y autocuidado destinados a prevenir complicaciones a causa de la hipertensión arterial. En el estudio realizado se muestra que la participación de los profesionales de enfermería es activo y eficiente.

Leyva R. et al, en su estudio "La capacidad de autocuidado en el control del paciente hipertenso en la unidad de Medicina Familiar del Instituto Mexicano del Seguro Social de Guanajuato", concluye que la capacidad de autocuidado fue buena en $73.4 \%$ de 
los hipertensos controlados, el $91.2 \%$ presentó un sistema familiar positivo muy similar a lo encontrado en nuestro estudio en el que el $62 \%$ de los pacientes cuentan con apoyo para cuidar su salud, el $86 \%$ mantiene buenas relaciones con sus familiares y el $78,00 \%$ tienen familiares que se preocupan por su salud, lo que se refleja en los resultados obtenidos en cuánto al estado de salud, el $38 \%$ tiene un estado de salud bueno, el $32 \%$ presenta un estado de salud excelente, el $16 \%$ regular y sólo el $14 \%$ tiene un estado de salud malo. En cuanto a los factores socioculturales, Leyva R., encontró que los factores socioculturales fueron buenos en $64.6 \%$, en nuestro estudio los factores socioculturales se caracterizan por tener un nivel alto $50 \%$. El $32 \%$ de los pacientes que participaron en nuestra investigación tiene estudios universitarios y el $24 \%$ tiene estudios de nivel técnico.

Florez M., en su tesis para optar el título de maestría en enfermería titulado "Asociación de los factores básicos condicionantes del autocuidado y la capacidad de agencia de autocuidado en personas con hipertensión arterial que asisten a consulta externa del Hospital Universitario la Samaritana Luz Carine Espitia Cruz", encontró que de los diez factores básicos condicionantes que plantea la Teoría de Orem, la edad, el estado de desarrollo, el estado de salud, los factores del ambiente y los patrones de vida tienen una asociación fuerte con la capacidad de autocuidado al arrojar valores de $p=<0.05$, confirmándose esta relación con nuestro estudio que demuestra que existe una relación directa y significativa entre el autocuidado y los factores individuales y socioculturales de los pacientes hipertensos del programa de hipertensión arterial de un hospital de Huancayo, para un nivel de significación de $\mathrm{a}=0,05$ lo que coincide con lo planteado por Flores.

\section{CONCLUSIONES}

Se demostró que existe una relación directa y significativa entre el autocuidado y los factores individuales y socioculturales de los pacientes hipertensos del programa de hipertensión arterial de un hospital de Huancayo, para un nivel de significación $a=0,05$ y $95 \%$ de nivel de confianza, con un coeficiente de correlación de " $r$ " Pearson = 0,647. Existe una correlación directa significativa entre los puntajes del autocuidado del paciente y los puntajes de los factores individuales de los pacientes hipertensos del programa de hipertensión arterial de un hospital de Huancayo, para 48 grados de libertad y $95 \%$ de nivel de confianza.

Se demostró que existe una correlación directa significativa entre los puntajes del autocuidado del paciente y los puntajes de los factores socioculturales de los pacientes hipertensos del programa de hipertensión arterial de un hospital de Huancayo, para un nivel de significación $5 \%$ y 48 grado de libertad.

\section{REFERENCIAS BIBLIOGRAFICAS}

1. Intriago V, Mévendez J. Autocuidado de pacientes hipertensos del club de corazones Valientes Hospital Naval de Guayaquil. Ecuador, 2010.

2. Colman J,Participación del profesional de enfermería en la promoción de hábitos de salud y el autocuidado destinados a prevenir complicaciones por hipertensión arterial Paraguay 2013".

3. Leyva R, Venegas O, Mendel A. La capacidad de autocuidado en el control del paciente hipertenso en la unidad de Medicina Familiar del instituto Mexicano del Seguro social de Guanajuato. México, 2011

4. Flórez M. Asociación de los factores básicos condicionantes del autocuidado y la capacidad de agencia de autocuidado en personas con hipertensión arterial que asisten a consulta externa del hospital universitario la Samaritana Luz Carine Espitia Cruz. Colombia, 2012.

5. Vásquez E. Autocuidado para el manejo de Hipertensión Arterial. Programa Departamento de Medicina Preventiva, Institución Hospitalaria de cuarto nivel". Bogotá 2008.

6. Fanarraga J. Conocimiento y Práctica de Autocuidado Domiciliario en Pacientes Adultos con Hipertensión Arterial, en Consultorios Externos de Cardiología del Hospital Guillermo Almenara Irigoyen ESSALUD. Perú, 2013.

7. Rasmussen, R. Estrategia en la promoción de la salud cardiovascular. Ponencia presentada en el 1er congreso virtual de Cardiología en Argentina (online). [Consultado el 15 de agosto 2014] Disponible http://www.fac.org.ar/cvirtual/ cvirtesp/cientesp/ceesp/cem3901c/crasmuss/ crasmuss.htm

8. De Reales, E. Autocuidado como estrategia para la prevención de la enfermedad y promoción de la salud cardiovascular. El Salvador. Mayo 6, -9 de 2003.

9. Gallegos, E. Validez y confiabilidades de la versión en español de la escala: Valoración de las Capacidades de Autocuidado. En: Desarrollo Científico de Enfermería. Vol.6, № 9 (1998); p.261.

10. Teorías de enfermeriuns.blogspot.com/2012/06/ dorothea-orem.htm/.

11. Hipertensión Arterial. Consultado el 15 de agosto 2014. Disponible en WWW.dmedicina. com/enfermedades/enfermedades.../ hipertensiónarterial

12. Siguenza J. Monografías. Disponible en :jdrsoo3@gmail.com monografías. com>salud.

13. López, J. Disponible en:www.Scielo.org.co/scielo. php?script $=$ sci

14. Bologna, E. Estadística para psicología y Educación. Argentina, 2011.

15. Christensen, Howard. (1994). Estadística paso a paso, Trills, México, 1994.

16. Fernández, M. et al. Curso básico de psicometría. Buenos Aires, Argentina, 2009.

17. Hernández, R. et al. Metodología de la investigación. Colombia. McGraw-Hill 
Interamericana.Colombia, 1997.

18. Hernández, $R$ et al. Metodología de la investigación. McGraw-Hill Interamericana. Chile, 2010

19. Lind, D. et al Estadística para administración y economía. Bogotá. Colombia, 2004.

20. Kaplan R, Sacuzzo D. Pruebas psicológicas. Sexta edición. México, 2006.

21. Martín E, Quintín A. et al. Tratamiento estadístico de datos con SPSS. Madrid. 2008.

22. Quezada N. Estadísticas con PASW 18. Lima: Empresa editora Macro EIRL.2009.
23. Solis C. Estadística descriptiva. Huancayo: Ckef ediciones.2013.

24. Triola M. Estadística. Décima edición. Pearson Educación. México 2010.

Fecha de recepción: 09/12/14

Fecha de aprobación: 26/12/14

Correspondencia: Suasnabar Cueva Edith

Email: suase23@hotmail.com

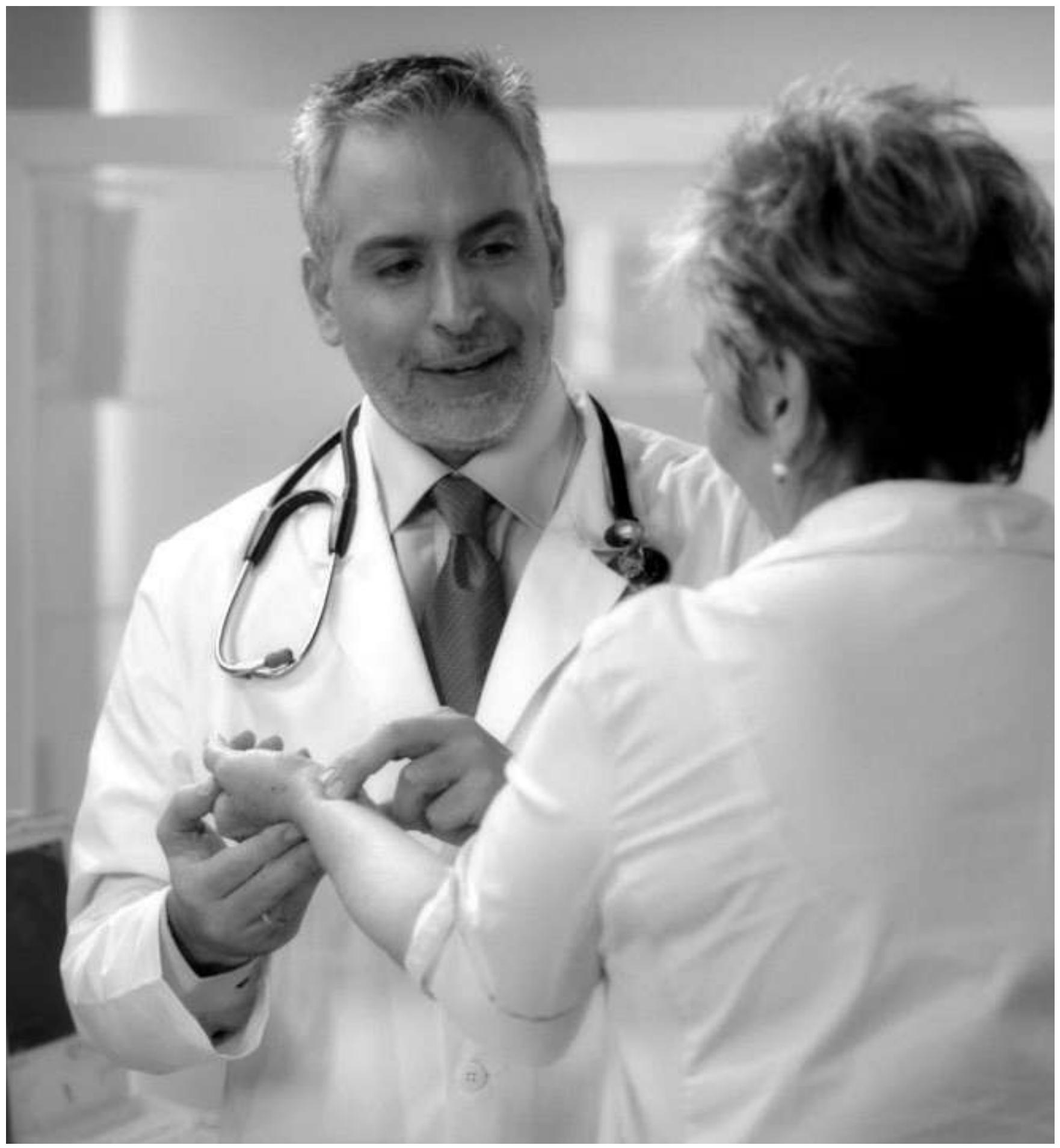

